\title{
The De-Central Bank in Decentralized Finance: A Case Study of MakerDAO
}

\author{
Martin Brennecke \\ FIM Research Center, \\ University of Bayreuth \\ $\underline{\text { martin.brennecke@ fim-rc.de }}$ \\ Benjamin Schellinger \\ FIM Research Center, \\ University of Bayreuth \\ benjamin.schellinger@fim-rc.de
}

\author{
Tobias Guggenberger \\ Project Group Business \& Information Systems \\ Engineering of the Fraunhofer FIT \\ tobias.guggenberger@fit.fraunhofer.de
}

\author{
Nils Urbach \\ Frankfurt University of Applied Sciences \\ \& Fraunhofer FIT \\ nils.urbach@fb3.fra-uas.de
}

\begin{abstract}
Countless decentralized finance (DeFi) applications of the past years have suffered from the high volatility and speculative behavior surrounding their underlying crypto assets. While the academic debate has been flourishing in these areas, Decentralized Autonomous Organizations (DAOs) have not received as much attention. This is the case even though they could offer an opportunity to solve some of the underlying problems of existing cryptocurrencies and ecosystems, for example, by providing lower volatility and, thus, exchange rate stability. This paper presents an economic analysis of the MakerDAO, a DAO in DeFi. In doing so, we use a single case study methodology based on existing resources and expert interviews. It also uses monetary theory instruments to provide researchers and developers with insights into how DAOs are governed. Further, it serves to illustrate how IS research may support the development of future IT artifacts aimed at offering the infrastructure for DeFi applications.
\end{abstract}

\section{Introduction}

On January 3rd, 2009, the first Bitcoin block ever created contained a direct reference to an article published on that very day in The Times, reading "Chancellor on the brink of second bailout for banks" $[1,2]$. While there is a discussion within the blockchain community on the question of whether the creation of the Bitcoin currency was a direct result of the financial crisis of 2007-2009, it stands to reason that distrust in financial intermediaries advanced Bitcoin's adoption. However, by the end of 2017, the native currency on the Bitcoin ledger increasingly turned into a speculative asset with high volatility [3]. Rather than becoming an alternative to traditional currencies, price fluctuations made it difficult to use Bitcoin as a medium of exchange [4].

Since then, over a decade has passed, and Bitcoin and other major cryptocurrencies such as Ether and XRP remain volatile, hence, inherently risky assets unfit for use as a means of payment [5]. Therefore, both researchers and practitioners recently began working on cryptography-based decentralized ecosystems that would use the underlying technology of the Bitcoin currency, namely blockchain, as the foundation for decentralized financial (DeFi) services. According to Chen [6] and Amler et al. [7], these DeFi services have the potential to enable entirely new business models and fundamentally transform modern finance, potentially reducing transaction costs, improving access to financial services, and lowering barriers to investment for entrepreneurs [8, 9].

However, the practical implementation of DeFi services relies on relatively stable cryptocurrencies that discourage short-term speculative behavior, enabling their productive use as a means of payment. One of the proposed solutions for high volatility in cryptocurrencies has been to implement stablecoins that aim to maintain a soft peg with real-world assets, such as fiat currencies, commodities, indices, or any combinations thereof [10]. Several stablecoins have already been implemented, successfully attracted considerable amounts of money, and are also used as a means of payment in countries suffering from high inflation and lack of political stability [11].

As of June $15^{\text {th }} 2021$, the stablecoin market is estimated to have a 110-billion-dollar capitalization, with Tether, USDCoin, Binance USD, and Dai claiming almost 90 percent of that market [12]. Hence, they are considered the dominant implementations of 
the concept of stablecoins, and as of June $15^{\text {th }} 2021$, the equivalent of more than 5 billion US dollars have been locked in Dai [12]. MakerDAO, considered to provide one of the advanced and prominent DeFi ecosystems, aims to use overcollateralization to generate said Dai stablecoin that may address many of the theoretical and practical challenges that other stablecoins have suffered from. As such, it could become an option for decentralized banking and is thus relevant to any organizations and individuals currently acting in a centralized financial system [13, 14]. However, from both a research and central bank perspective, MakerDAO opens the door to uncharted terrain in the analysis of IS and monetary theory.

However, there is little literature on MakerDAO. We aim at addressing this research gap by gaining and providing an in-depth and interdisciplinary understanding of fiat-pegged stablecoins in general and the MakerDAO ecosystem in specific. This lack of existing literature may result in risks for researchers and practitioners. We resolve this by offering novel and relevant insights in DeFi and central banking.

In doing so, our goal is threefold, as we want to (1) create a foundation for future research in $\mathrm{DeFi}$, stablecoins, and the MakerDAO ecosystem by collecting and consolidating previously unstructured resources, (2) advance the theoretical understanding of the real-world applications of blockchain technologies in IS research, as well as (3) foster the academic exchange between researchers in IS and monetary theory to avoid past mistakes and increase the viability of these IT artifacts.

Following the single case study approach of Yin [15], we will extend the existing research in blockchain-related systems science and monetary theory. By building on existing IS research, this paper refers to academic insights into blockchain, DeFi, and stablecoins. The theoretical background of this paper will be based on the monetary regimes surrounding the interwar gold exchange standards and the Bretton Woods financial system [16], as this time in economic history shares several key similarities with the ongoing challenges in DeFi. Consequently, we address a revelatory case that may serve as an interdisciplinary foundation for future longitudinal analysis of the MakerDAO ecosystem but also investigates a typical case as MakerDAO is currently the prominent representative of DeFi by total value locked [17].

\section{Theoretical background}

\subsection{Economics and monetary policy}

To motivate and understand the reasons for the popularity of cryptocurrencies and the need for exchange rate stability in monetary systems, this section provides an overview of the Bretton Woods system, its aims and prerequisites, its mechanisms, and execution, as well as its collapse and learnings.

As a result of World War II and the abrupt halting of production for the war, the international system of trade and commerce had taken a significant hit, and trust between many developed nations was severely damaged [18]. During this time, currencies across the developed world were highly volatile [19], and trade between nations became unsustainably costly as there was a high level of currency and exchange rate risk involved in any transactions with other nations [20]. Economists and heads of state alike knew that both citizens and organizations require stable currencies and exchange rates if trade between nations was to take place again. Hence, delegates from all Allied countries convened for the United Nations Monetary and Financial Conference at the Mount Washington Hotel for the Bretton Woods Conference [21].

This conference aimed to establish an international exchange rate system that would facilitate trade among all participating nations by reducing the risk caused by the high volatility of exchange rates [22]. There were two competing proposals for how the system of international trade could be designed [23]. The American proposal, also known as the White-Plan, was built on quota-based funds in which international accounts would be balanced using special drawing rights [24]. Under this plan, the entire international trade system was based on the US dollar, which in turn rested on the gold standard under which the US dollar was fully backed by gold, and both assets could be freely exchanged by individuals, organizations, and governments [25]. For about three decades, this system was able to reduce exchange rate risks but ultimately collapsed after the US government abolished the gold standard, officially turning the US dollar into a fiat currency [26].

Specifically, this time was shaped by uncertain political environments, high volatility, and exchange rate instabilities [27, 28]. Furthermore, many of the motivators for financial reform are comparable with the post-War economic environment on both sides of the Atlantic, considering an unstable international trade system, high exchange rate risks and the prisoner's dilemma involved in making free trade a welfare-optimizing strategy choice [29].

The Bretton Woods system was a fixed exchange rate regime in which several currencies were pegged to the US dollar, and the US dollar was backed by gold held in US reserves. The consequences of the failure of this system ultimately led to numerous advances in research of monetary theory but has not yet entered the discussion surrounding the development of IT artifacts 
aiming to achieve equivalent or similar goals. We expect that some of the problems surrounding early cryptocurrencies could be avoided if developers, organizations, and end-users had learned from past challenges and failures of centralized banking. Thus, the Bretton Woods system serves as a promising object of comparison for the analysis of the MakerDAO ecosystem, as it was established to reduce currency and exchange rate risks on markets with multiple, non-trusting actors over prolonged periods.

\subsection{Blockchain, DeFi, and stablecoins}

In 2008, Satoshi Nakamoto published their whitepaper 'Bitcoin: A Peer-to-Peer Electronic Cash System,' which laid the foundation for the cryptocurrency Bitcoin [30]. While Bitcoin itself was initially considered revolutionary, researchers soon focused on its underlying technology: the blockchain, which enabled business models and processes that went far beyond their use as an infrastructure technology for a decentralized currency [31, 32]. While Bitcoin has previously been referred to as the new gold [33], some researchers claimed that Bitcoin de facto lacks an intrinsic value [3]. Klein et al. [33] also found that Bitcoin behaves asymmetrically to many primary precious metals, despite the energy required to mine Bitcoin or execute transactions [34].

Building on the ideas of Nakamoto, open-source developers, foundations, and companies soon moved into the development of alternative cryptocurrencies, making various cryptocurrency implementations available to the public. The Swiss-based Ethereum Foundation developed the Ethereum network, allowing users to execute program code (smart contracts) implemented on the blockchain [35]. In the beginning, smart contracts were primarily used to replicate real-world assets, processes, and contracts on the blockchain. Use cases during the initial stages included, among others, supply chain management [36], voting mechanisms or systems [37], and crossborder payments [38]. Recently, it opened the field of DeFi for academic research and practical application.

According to Zetzsche et al. [39], DeFi may be defined by its incorporation of decentralization, distributed ledgers, smart contacts, disintermediation, and open banking. As such, it stands in contrast to both traditional finance and centralized banking as it could allow for a democratization of finance and monetary policy by broadening the opportunities for access, removing potential gatekeepers, and placing responsibility for policy decisions in the hands of the system's actual users [40, 41]. Thereby, DeFi is increasingly relevant for areas such as international development, reserve banking, political governance, and business applications [9, 42, 43].

However, one of the main problems of early examples of DeFi was that their underlying cryptocurrencies often suffered from high volatility and exchange rate risks [44]. Consequently, they were attractive to speculative investors but fell short of their promise to create an infrastructure for decentralized banking. Considering this problem in the productive implementation of decentralized monetary systems, developers turned their attention to developing cryptocurrencies that are less attractive as speculative assets and would maintain a certain amount of internal and external stability.

These considerations resulted in the development of stablecoins. Despite their potential to drive innovations in developed financial systems, one of the impactful uses of DeFi services will likely occur in regions where significant numbers of people are either unbanked or underbanked [45]. In those regions specifically, DeFi could enable a decentralized monetary system independent from the influence of non-competitive actors and speculative shareholders.

Stablecoins are "crypto-native assets that are stable in value and maintain a stable rate of exchange" [46, p. 1]. Recently, Lipton et al. defined them as "a digital unit of value with the following properties: (i) It is not a form of currency, (ii) it can be used without any direct interaction with the issuer, (iii) it is tradable on a secondary market and has a low price volatility in terms of a target quote currency" [47, p. 5].

$\mathrm{Gu}$ et al. [46] broadly categorize stablecoins based on whether they are collateralized on or off the ledger. Nevertheless, further differentiations are possible concerning (i) the type and amount of the collateral, (ii) the stabilization or governance mechanisms, (iii) the price information provider, and (iv) the asset that the respective stablecoin is pegged to [10].

First, concerning the type and amount of collateral, different implementations of stablecoins may choose to hold a full reserve or partial reserve; they may also overcollateralize or avoid collateralization entirely. If they opt for collateralization, they may hold reserves of existing fiat money, tokenized commodities, cryptocurrencies, or a combination thereof. Second, they have the option of choosing a mechanism or use case such as a reserve of backed assets, a dual coin, an algorithm, or leveraged loans. Third, they may select oracles, voting systems, or trades to govern price information. Forth, they may be pegged to a fiat currency, tokenized commodity, an index, or combination thereof [10]. Dai serves as the stablecoin for the DeFi application MakerDAO. It is based on the Ethereum blockchain, initially relied on Ether, and is pegged to the US dollar. 
Additionally, it uses leveraged loans and median oracles to adjust prices and supply.

While Tether and the USD Coin are partially collateralized, the MakerDAO ecosystem features an over-collateralization mechanism [10]. Due to these features, MakerDAO is one of the widely adopted $\mathrm{DeFi}$ protocols [12], making an analysis of the ecosystem critical and revelatory. However, prior to this analysis, it is important to first define DAOs.

\subsection{Decentralized autonomous organizations}

There are two fundamentally different schools of thought in the governance of blockchains and blockchain-based applications: a techno-determinist one and a critical one [48, 49], with little room in between. While the techno-determinist view is marketdriven and might fail to spot and adapt to the realworld requirement of social organizations, the critical view tends to recognize these requirements but primarily focuses on centralized institutions and how they could solve these downsides [49]. Consequently, there is no universally accepted definition of what constitutes a DAO but rather several broadly recognized definitions that share a hand full of mutual elements. From the perspective of the blockchain community, the definitions by Luis Cuende [50] and Vitalik Buterin [51] are the prominent ones, while academic researchers have recently converged upon the definitions by Jentzsch [52] and Chohan [53].

According to Cuende, "A DAO is an internetnative entity with no central management which is regulated by a set of automatically enforceable rules on a public blockchain, and whose goal is to take a life of its own and incentivize people to achieve a shared mission." [50] Buterin argued in 2013 that a DAO is "a virtual entity that has a certain set of members or shareholders which [...] have the right to spend the entity's funds and modify its code." Jentzsch [52, p. 1] proposed that DAOs are "organizations in which (i) participants maintain direct real-time control of contributed funds and (ii) governance rules are formalized, automated and enforced using software," while Chohan argues that "a DAO, is an organization that is run through rules encoded as computer programs called 'smart contracts." [53, p. 1]

However, the latest discussion on the definition of DAOs, to date, has been authored by Hassan and De Filippi: "A DAO is a blockchain-based system that enables people to coordinate and govern themselves mediated by a set of self-executing rules deployed on a public blockchain, and whose governance is decentralized (i.e., independent from central control)." [54, p. 1] After defining DAOs as such, we identified the method as well as the data collection.

\section{Method and data collection}

Over the past decade, researchers have been primarily interested in understanding blockchain itself and the potential areas in which it may be productively used [35, 43]. As a result, they have designed and evaluated IT artifacts, conceptualized potential use cases, and discussed the effects blockchain might have on organizations and individuals [55]. This paper builds on these insights in order to analyze the MakerDAO ecosystem using a single case study, following the recommendations by Yin [15], which will be complemented by insights into case study research in the context of blockchain applications, such as those identified by Treiblmaier [56].

The primary object of investigation in this single case study is the MakerDAO ecosystem as an example of a DeFi-based IT artifact. While blockchain-based cryptocurrencies have been around for over a decade now, their real-world applicability has been limited due to the involved risks and speculation [57]. By recognizing some of the obstacles that previous monetary regimes have faced and how they were overcome, researchers and developers in IS research can further improve existing DAOs and DeFi systems.

Following the research process for single case studies as proposed by Yin [15] and applied under similar circumstances by Miscione et al. [58], we planned, designed, prepared, collected, analyzed, and shared their ideas, framework, and results. Consequently, our research is based on MakerDAOs documentation, forums, developer boards, as well as implemented and proposed code snippets. A list of sources used for this study may be found in Table 1 .

Based on the information gathered during this analysis of existing resources on MakerDAO and research on the Bretton Woods system, central properties of the MakerDAO ecosystem and the Bretton Woods system are derived and compared. The resulting monetary standards and comparisons are then validated using expert interviews with individuals highly knowledgeable in the fields of DeFi and DAOs, as well as economics and monetary theory.

As per usual in exploratory and qualitative research, we use the information available in a variety of different sources $[56,57]$ to accurately understand the ever-evolving MakerDAO ecosystem and its internal debates and future directions development. This includes information available via Scopus, the Web of Science, and Google Scholar using 'MakerDAO,' 'Dai,' and 'decentralized autonomous organization' as central search queries. We also rely on the available literature on the Bretton Woods monetary regime in general, the gold coin, the gold bullion, and the gold exchange standard. 
Following this review of the existing literature, we individually and independently analyzed the sources presented in Table 1. The information gathered there was then supplemented with the previously mentioned secondary sources covering the MakerDAO ecosystem from an academic perspective. Many of these papers were theory-, development-, or governance-oriented and failed to draw direct links to potentially similar economic and monetary regimes in the past and present. Additionally, the literature also provided insights into the economic and monetary policy during and after the Bretton Woods era.

\section{Table 1. Sources and resources}

\begin{tabular}{|l|r|}
\hline MakerDAO Whitepaper [59] & 15 pages \\
\hline MakerDAO Documentation [60] & 423 pages \\
\hline MakerDAO 101 [61] & 68 pages \\
\hline MakerDAO Developer Guides [62] & 398 pages \\
\hline MakerDAO FAQ (legacy) [63] & 109 pages \\
\hline MakerDAO Forum [64] & 50 pages \\
\hline
\end{tabular}

In a second step, we entered an exchange phase in which we discussed, evaluated, and structured the results from our independent research. In doing so, we constructed a framework in which we compared the MakerDAO ecosystem with the gold bullion standard and the gold exchange standard using seven specific dimensions within the two groups of governance and value. Based on this, they were able to evaluate which of the seven dimensions in the MakerDAO ecosystem closely matched the different standards.

The results up to this point were then presented during a research colloquium with about a dozen researchers in business and IS, including blockchain researchers, thereby receiving first constructive feedback on the results. This feedback was then integrated into the overall framework, which was consequently adapted. Using semi-structured interviews with additional experts in both DeFi and economics, we then iteratively adjusted and improved the initial artifact. Our sample of experts represented a wide range of topics in DLT, Blockchain, and economics, such as DeFi, traditional public finance, and monetary theory, with relevant experience between two and six years. A condensed overview of the experts is presented in Table 2.

The interviews consisted of an opening, an informed consent disclaimer, a discussion of the interviewees' work history, the presentation of our research results, the evaluation of our research results, and the closure of the interview itself. Further information on our research process, including the structure of the interviews and the central insights gathered are listed in the online appendix [65].

\section{Table 2. Condensed overview of experts}

\begin{tabular}{|l|l|r|r|}
\hline & Background & Field & Relev. Experience \\
\hline 1 & DLT \& DeFi & Research & 3 years \\
\hline 2 & DLT \& DeFi & Industry & 2 years \\
\hline 3 & DLT & Industry & 2 years \\
\hline 4 & Economics & Research & 3 years \\
\hline 5 & Economics & Research & 6 years \\
\hline 6 & Economics & Industry & 4 years \\
\hline
\end{tabular}

This process aimed to refine our understanding of the MakerDAO ecosystem, evaluate our illustration of the MakerDAO ecosystem, and evaluate our artifact to demonstrate the similarities of MakerDAO with other monetary regimes. It also allowed us to identify implications that a system such as MakerDAO could have in the areas of institutional governance, insurance economics, and taxation. However, an understanding of these implication relies on a detailed understanding of the MakerDAO ecosystem.

\section{The MakerDAO ecosystem}

The MakerDAO Foundation set out to create an unbiased global financial system that could improve both (decentralized) finance and monetary policy [59]. For this purpose, they implemented the stablecoin Dai, the governance token MKR, and a governance system to gain access to and manage the entire ecosystem without relying on intermediaries $[59,66]$.

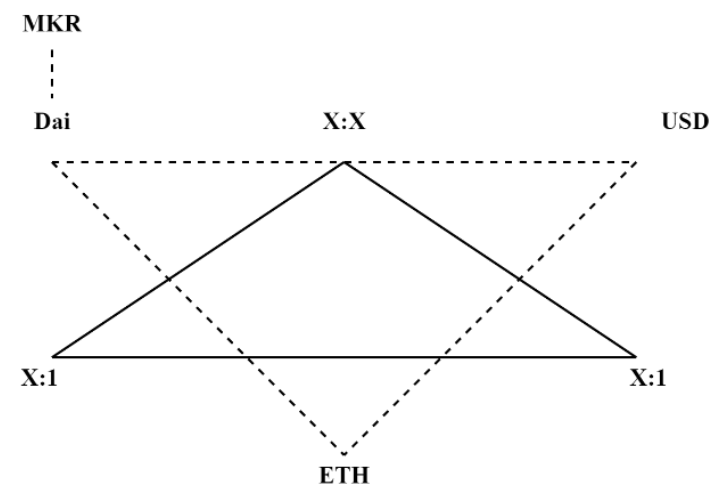

Figure 1. Exchange rates between the US dollar, Dai, and its collateral vault

Using complex smart contract structures [67] to enable the mechanism demonstrated in Figure 1, the stable coin Dai was created to maintain a constant exchange rate of $X: X \equiv 1: 1$ to the US dollar, which it aims to achieve by relying on an over-collateralization with multiple different collaterals in a vault [59].

Once the collateralization rate of, i.e., $150 \%$ has been exceeded, a user may take out a Dai loan for the sake of leverage and/or liquidity. As soon as the value 
of the backing asset falls below the liquidation rate of, i.e., $150 \%$, its underlying vault, and its contents will be liquidated [68]. Hence, users have been aiming for higher collateralization rates and collateral diversification to avoid an auction of their assets in case of significant market shocks and volatilities. Until recently, the system relied solely on Ether as collateral but has expanded to other cryptocurrencies.

Multi-collateral vaults (MCVs are usually aimed at risk reduction via a portfolio diversification [69] and, in the case of MakerDAO, are currently undergoing further development. For collateral types to be added, they have to be proposed, evaluated, and approved by governance token (MKR) holders [70].

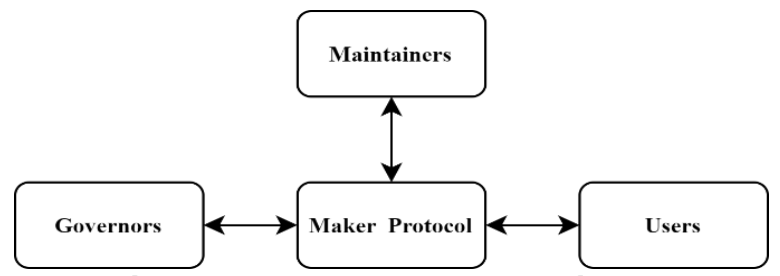

Figure 2. Agents and actors in the MakerDAO ecosystem based on [61]

As shown in Figure 2, we discovered that the MakerDAO ecosystem consists of seven types of actors that may be categorized into three different groups: Users, Maintainers, and Governors. Users are either holders of Dai or owners of vaults. Maintainers may be developers, oracles, or keepers. Governors are either MKR holders or members of risk teams. These roles are either staffed by individuals, groups of individuals or machines, and are not mutually exclusive, meaning that a Dai holder could also be a vault owner, developer, and risk team member.

The decision-making process in the MakerDAO ecosystem relies on the cooperation of risk teams and MKR holders. While the risk teams act primarily as advisors, the MKR holders can vote on any technical and non-technical changes within the ecosystem. While some changes are made regarding the off-chain environment, others concern the on-chain governance.

Each MKR holder is able to propose a governance change by executing a proposal contract. MKR holders are then polled to ensure that a given proposal is viable. The proposal will either be accepted or denied depending on whether it receives a majority of MKR holder votes or not. According to the MakerDAO whitepaper [59], MKR holders may vote on adding to collateralization asset types, setting and adjusting risk parameters, setting the Dai Savings Rate, selecting oracle feeds, shutdown the ecosystem in case of emergency, or perform updates [71, 72].

The MakerDAO ecosystem is governed via onand off-chain mechanisms. The on-chain governance primarily concerns polls and executive votes in the Maker Forum's voting list. While the former captures the community's general attitude towards a draft proposal to create a consensus, the latter executes technical changes. On-chain governance is facilitated by three central smart contracts. Chief is a smart contract that allows MKR holders to select a primary contract to be executed using their voting privileges. A pause is a smart contract that allows MKR holders to enforce a delay in executing specific calls. A spell is a smart contract that may be utilized to set technical constraints such as system parameters. Also, off-chain governance refers to any discussions among community members outside of the on-chain mechanisms. Hence, off-chain governance consists primarily of forum signal threats, forum polls, and blog entries by the community. The primary challenge for the MakerDAO community lies in implementing the infrastructure for MCVs and further decentralizing the overall governance structure of its ecosystem.

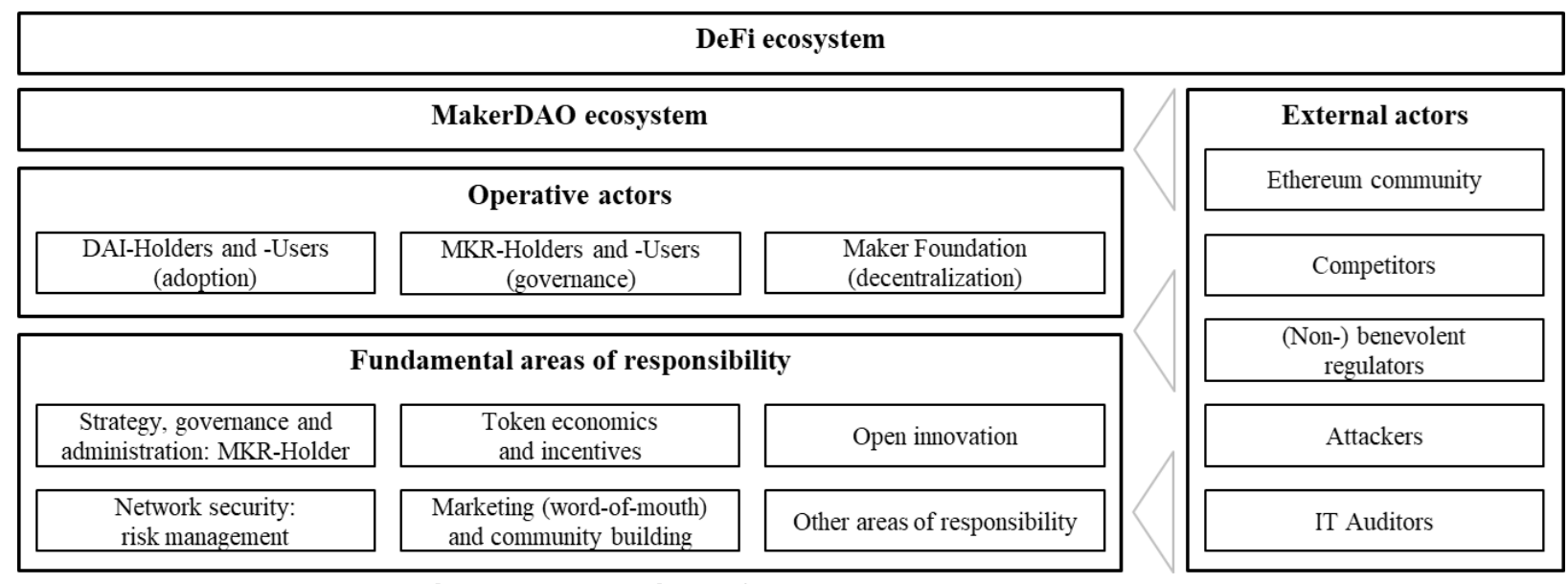

Figure 3. Overview of the MakerDAO ecosystem 
Nevertheless, the MakerDAO ecosystem does not solely rely on the stablecoin (Dai) and governance token (MKR), as well as its immediate operative actors but also on external actors and agents. Figure 3 aims to present and classify these additional internal and external stakeholders. It also indicates the areas of responsibility for the system and its stakeholders.

\section{Towards DAO-based currency systems}

A DAO-based currency system could contribute to solving some of the primary challenges of banking in the $21^{\text {st }}$ century, as its strengths and opportunities could lie in its ability to form an infrastructure for financial inclusion, participation in investments, interoperability, and disintermediation [6].

Across the globe, it is estimated that around 1.7 billion adults are unbanked, hence disconnecting them from the global financial markets [45]. Second, participation in investment could be made available to those same individuals and organizations that are currently unbanked, thus enabling them to incur debt to create value in the future. Third, it could lead to an increase in interoperability of cryptocurrencies and other assets. As oracle teams are developing price oracles for various assets, users can use several different collateralization types to take out a stablecoin loan. However, it is unlikely that blockchain-based organizations will replace existing centralized institutions such as central or commercial banks [73].

In the history of monetary policy, an evolution occurred that resulted in establishing a goodsexchange economy, the gold coin standard, the gold bullion standard, the gold exchange standard, and a floating exchange rate. While previous research suggests similarities between Bitcoin and the gold coin standard $[74,75]$, and due to a limited supply and the fact that resources are required for mining it, there has been little research into whether DeFi applications could replicate further advances in monetary theory to create a decentralized global financial system that enables financial transactions with little to no speculation and volatility.

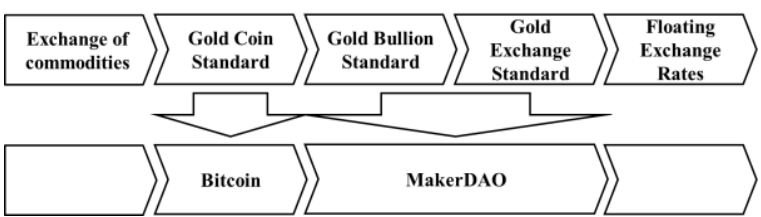

\section{Figure 4. Parallels between Bitcoin, MakerDAO, and the development of monetary regimes}

As shown in Figure 4, we argue that the MakerDAO ecosystem partially meets the configurations of the gold bullion standard and the gold exchange standard, thus allowing a comparison that places MakerDAO in between both standards. As free floating exchange rates are currently too volatile in $\mathrm{DeFi}$, it also remains to be identified how future DeFi artifacts may enable them reliably.

As illustrated on the left side of Table 3, there are two different categories of dimensions with four dimensions each. While the dimensions of stabilization, mechanism, backed by, and collateral fall into the value category, the dimensions of policy, primary actors, secondary actors, and decisions falls into the governance category. The two categories are closely intertwined as an improvement of the governance of a system increases its value, while an increase in a system's value necessitates a better governance for the system to run more efficiently.

\begin{tabular}{|c|c|c|c|c|}
\hline & Dimension & Gold Bullion Standard & MakerDAO & $\begin{array}{r}\text { Gold Exchange Standard } / \\
\text { Fixed Exchange Rate Regime }\end{array}$ \\
\hline \multirow{4}{*}{$\stackrel{\stackrel{g}{\Xi}}{\stackrel{一}{\nu}}$} & Stabilization & Fiat currency is stabilized using gold bullion & $\begin{array}{l}\text { Individual risk preference via collateralization } \\
\text { rates and stabilization fees; transparency }\end{array}$ & $\begin{array}{l}\text { US dollar is stabilized using gold bullion } \\
\Rightarrow>3 \text { rd country currency }\end{array}$ \\
\hline & Mechanism & Melting and printing & $\begin{array}{l}\text { Smart contract enabled locking and receiving; } \\
\text { community sets rates and fees }\end{array}$ & $\begin{array}{l}\text { Melting, printing and exchanging; central banks } \\
\text { peg exchange rates }\end{array}$ \\
\hline & Backed by & Value of gold; trust in government; tax revenue & $\begin{array}{l}\text { Trust in code; trust in community; trust in MKR- } \\
\text { Holders; trust in US dollar; trust in collaterals }\end{array}$ & $\begin{array}{l}\text { Value of gold; trust in US government; trust in } \\
\text { central banks }\end{array}$ \\
\hline & Collateral & Gold bullion (gold reserve) & $\begin{array}{l}\text { Currently: selection of different } \\
\text { cryptocurrencies; Subsequently: other crypto } \\
\text { assets, such as NFTs }\end{array}$ & Gold bullion (gold reserves) and US dollar \\
\hline$\hat{i}$ & Implications & \multicolumn{3}{|c|}{ Improved governance creates higher value and higher value necessitates better governance. } \\
\hline \multirow{4}{*}{ } & Policy & $\begin{array}{l}\text { Central bank or Federal Reserve decide on } \\
\text { amount printed }\end{array}$ & $\begin{array}{l}\text { MKR-Holders decide on policy proposals } \\
\text { submitted by the entire community; the process } \\
\text { is public and invites open innovation }\end{array}$ & $\begin{array}{l}\text { Bretton Woods and subsequent national } \\
\text { agreements }\end{array}$ \\
\hline & \begin{tabular}{|l} 
Primary \\
Actors
\end{tabular} & $\begin{array}{l}\text { Government; central banks; Federal Reserve; } \\
\text { commercial banks }\end{array}$ & $\begin{array}{l}\text { Maker Foundation; developers; holders of } \\
\text { stablecoin Dai or governance token MKR }\end{array}$ & $\begin{array}{l}\text { Government; Federal Reserve; central banks; } \\
\text { and IMF/World Bank }\end{array}$ \\
\hline & \begin{tabular}{|l} 
Secondary \\
Actors
\end{tabular} & Users/citizens & $\begin{array}{l}\text { Interacting users; regulators; attackers; } \\
\text { cryptocommunity }\end{array}$ & Central banks; governments \\
\hline & Decisions & $\begin{array}{l}\text { Central issuer of currency dictates monetary } \\
\text { policy }\end{array}$ & MKR holders; aquirable vote shares & Basic votes; and IMF/World Bank \\
\hline
\end{tabular}

Table 3. Comparison of the MakerDAO with the gold bullion and gold exchange standard 
Some of the relevant results of our interviews beyond those listed in Table 3 may be clustered in a total of three groups covering (i) the advantages of free floating interest rates, (ii) the potential broad about by a community-agreed upon pricing of non-fungibile tokens (NFTs) in modern taxation schemes, and (iii) the importance of the size of the overall system by value locked and active use.

The gold exchange standard and a fixed exchange rate regime might be crucial in enabling different cryptocurrencies to converge towards each other, easing the risk of a future move towards floating exchange rates. However, as MakerDAO does not yet meet all theoretical requirements of the gold exchange rate standard, it will be unable to move towards floating exchange rates in the foreseeable future. Hence, future development efforts should focus on the eight dimensions indicated in Table 3, while also being aware of the implications that hold between them.

Additionally, limitations may include the fact that there is limited oversight, limited powers for users, and a lack of insurance against a loss of assets within the network [44]. Because many centralized banking systems offer a deposit guarantee, this will keep more traditional investors from using MakerDAO as means of payment. This issue is further worsened since it would be precisely those traditional investors that could further stabilize the overall ecosystem [42].

Despite limitations, MakerDAO partially achieved its goals, which places it in the pole position regarding future advances towards the gold exchange standard and floating exchange rates. From the internal perspective, these achievements include (multi-asset) collateralization [59], (partial) decentralization [72], and lower volatility [12]. From the external perspective, MakerDAO was able to implement a comparatively stable cryptocurrency.

\section{Conclusion}

Our research contributes to IS researchers' limited but growing practical understanding of DeFi, stablecoins, MakerDAO and their interdisciplinary conceptualization at the intersection of research in IS research and monetary theory. Additionally, we have contributed to advancing our disciplines' theoretical understanding of the real-world applications of blockchain technologies and their challenges. Our analysis also enables practitioners and researchers to work on some of the shortcomings of MakerDAO compared to other monetary regimes.

These shortcomings may be retrieved from the extent to which MakerDAO matched the gold exchange standard in the dimensions of stabilization, stability mechanism, backed by, and collateral. In the governance category, they may be retrieved from the dimensions of policy, primary actors, secondary actors, and decision-making processes. It is also crucial to consider the co-dependency of value and governance, because a better governance will improve the ecosystems' overall value, which will necessitate an improved governance.

We evaluated the MakerDAO ecosystem based on both literature and expert interviews and outlined the foundation for a disintermediated global financial system using the concept of a DAO. In addition, we classify the MakerDAO ecosystem and provide nine areas for future research in $\mathrm{DeFi}$, enabling a better understanding of MakerDAO as phenomenon in DeFi.

The practical limitations of our research are threefold as (1) there is very limited peer-revied research available on MakerDAO, (2) only a limited number of experts could be consulted, and (3) MakerDAO is a quickly evolving ecosystem in which information has a short-lived half-life. Therefore, research should also aim at improving insights into MakerDAO, consult additional experts in $\mathrm{DeFi}$, and continue to observe the MakerDAO community to catch updates regarding its value- or governance-driving dimensions.

Specifically, future research in DAO value should focus on the expansion of collateralization types to real-world assets such as NFTs as this might unlock future lines of research at the intersection of finance, taxation, and transparency. Furthermore, future research in DAO governance could focus on policy, as correctly setting a DAO's policies could enable a more open, innovative, and democratic financial system.

Outside of MakerDAO, we are aware that our framework will only be transferrable if the aims and at least part of the governance mechanisms of the object of comparison aligns with those of MakerDAO. If transferred to an entirely different DeFi application, we expect the dimensions in the field of value to change while the dimensions in the field of governance could be equally relevant. Lastly, the connection between value and governance will likely hold for other DeFi applications.

As of now, MakerDAO does not appear to replicate the primary goals of a central bank, namely price stability, support of economic activity, and a reduction in unemployment, but provides a valuable step towards a more stable DeFi ecosystem. Once its collateral-backed stablecoin ecosystem has been able to replicate the gold exchange standard in a decentralized and economically sustainable manner, research may also focus on how cryptocurrencies could move, from a hard- or soft-peg to another currency or asset, towards a free-floating exchange rate. 


\section{References}

[1] Bitcoin Explorer. Blockchain Genesis Block; 2021. Available from: URL: https://bitcoinexplorer.org/tx/4a5e1e4baab89f3a3251 8a88c31bc87f618f76673e2cc77ab2127b7afdeda33b.

[2] Elliot F, Duncan G. Chancellor Alistair Darling on brink of second bailout for banks: Billions may be needed as lending squeeze tightens. The Times 2009.

[3] Baur DG, Hong K, Lee AD. Bitcoin: Medium of exchange or speculative assets? J. Int. Financial Mark. Inst. Money 2018; 54: 177-89 [https://doi.org/10.1016/j.intfin.2017.12.004]

[4] Baur DG, Dimpfl T. The volatility of Bitcoin and its role as a medium of exchange and a store of value. Empir. Econ. 2021: 1-21 [https://doi.org/10.1007/s00181-020-01990-5]

[5] Katsiampa P. An empirical investigation of volatility dynamics in the cryptocurrency market. Research in International Business and Finance 2019; 50: 322-35 [https://doi.org/10.1016/j.ribaf.2019.06.004]

[6] Chen Y. Decentralized Finance: Blockchain Technology and the Quest for an Open Financial System. SSRN Journal 2019 [https://doi.org/10.2139/ssrn.3418557]

[7] Amler H, Eckey L, Faust S, Kaiser M, Sandner P, Schlosser B. DeFi-ning DeFi: Challenges \& Pathway; 2021.

[8] Grigo J, Hansen P, Patz A, Wachter V von. Decentralized Finance: A new Fintech Revolution?; The Blockchain Trend explained: Bitkom; 2020.

[9] Schär F. Decentralized Finance: On Blockchain- and Smart Contract-based Financial Markets; 2020.

[10] Moin A, Sekniqi K, Sirer EG. SoK: A Classification Framework for Stablecoin Designs. In: Bonneau J, Heninger NA, editors. Financial cryptography and data security: 24th International Conference, FC 2020. Cham: Springer 2020; 174-97.

[11] Kshetri N, Voas J. Blockchain in Developing Countries. IT Prof. 2018; 20(2): 11-4 [https://doi.org/10.1109/MITP.2018.021921645]

[12] CoinMarketCap. Top Stablecoin Tokens by Market Capitalization; 2021. Available from: URL: https://coinmarketcap.com/view/stablecoin/.

[13] Bullmann D, Klemm J, Pinna A. In search for stability in crypto-assets: Are stablecoins the solution?; 2019. Occasional Paper Series 230.

[14] Sidorenko EL. Stablecoin as a New Financial Instrument. In: Ashmarina SI, Vochozka $\mathbf{M}$, Mantulenko VV, editors. Digital Age: Chances, Challenges and Future. Cham: Springer 2020; 630-8.

[15] Yin RK. Case Study Research and Applications: Design and Methods. Sixth edition. LA: SAGE 2018.

[16] Kugler P, Straumann T. International Monetary Regimes: The Bretton Woods System. In: Battilossi S, Cassis Y, Yago K, editors. Handbook of the History of Money and Currency. Springer 2020; 665-85.

[17] DeFi Pulse. DeFi Leaderboard; 2021. Available from: URL: https://defipulse.com/.

[18] van Dormael A. Bretton Woods: Birth of a Monetary System. Palgrave Macmillan 1978.
[19] Bayoumi T, Eichengreen B. Exchange rate volatility and intervention. J. Int. Econ. 1998; 45(2): 191-209 [https://doi.org/10.1016/S0022-1996(98)00032-4]

[20] Rose AK. One money, one market: the effect of common currencies on trade. Economic Policy 2000; 15(30): [https://doi.org/10.1111/1468-0327.00056]

[21] Lamoreaux N, Shapiro I. Introduction. In: Lamoreaux N, Shapiro I, editors. Bretton Woods Agreements. Yale University Press 2019; 1-18.

[22] Eichengreen B. The Monetary Role of Gold as the Original Sin of Bretton Woods. In: Lamoreaux N, Shapiro I, editors. Bretton Woods Agreements. Yale University Press 2019; 38-55.

[23] Steil B. The battle of Bretton Woods: John Maynard Keynes, Harry Dexter White, and the making of a new world order. Princeton University Press 2013.

[24] Cameron D. Special Drawing Rights. International Journal 1981; 36(4): 713-31 [https://doi.org/10.1177/002070208103600402]

[25] Proceedings and Documents of the United Nations Monetary and Financial Conference 1944.

[26] Bordo MD, Eichengreen BJ. A Retrospective on the Bretton Woods system: Lessons for international monetary reform. University of Chicago Press 1993.

[27] Cogley T, Sargent TJ. Measuring Price-Level Uncertainty and Instability in the United States, 18502012. Rev. Econ. Stat. 2015; 97(4): 827-38 [https://doi.org/10.1162/REST_a_00498]

[28] Grilli V, Kaminsky G. Nominal exchange rate regimes and the real exchange rate. J. Monet. Econ. 1991; 27(2):

$191-212$ [https://doi.org/10.1016/0304-3932(91)90041-L]

[29] Goldstein J, Gowa J. US national power and the postwar trading regime. World Trade Review 2002; 1(2) [https://doi.org/10.1017/S1474745602001131]

[30] Nakamoto S. Bitcoin: A Peer-to-Peer Electronic Cash System; 2008.

[31] Mattila J. The Blockchain Phenomenon The Disruptive Potential of Distributed Consensus Architectures; ETLA Working Papers. Helsinki: The Research Institute of the Finnish Economy (ETLA); 201638.

[32] Memon M, Hussain SS, Bajwa UA, Ikhlas A. Blockchain Beyond Bitcoin. In: Blockchain Beyond Bitcoin; 82018. IEEE; 29-34.

[33] Klein T, Pham Thu H, Walther T. Bitcoin is not the New Gold. Int. Rev. Financial Anal. 2018; 59: 105-16 [https://doi.org/10.1016/j.irfa.2018.07.010]

[34] Vries A de. Bitcoin's Growing Energy Problem. Joule 2018; 2(5): 801-5 [https://doi.org/10.1016/j.joule.2018.04.016]

[35] Schlatt V, Schweizer A, Urbach N, Fridgen G. Blockchain: Grundlagen, Anwendungen und Potenziale; 2016.

[36] Saberi S, Kouhizadeh M, Sarkis J, Shen L. Blockchain technology and its relationships to sustainable supply chain management. Int. J. Prod. Res. 2019; 57(7) [https://doi.org/10.1080/00207543.2018.1533261]

[37] Kshetri N, Voas J. Blockchain-Enabled E-Voting. IEEE Softw. 2018; 35(4): 95-9 [https://doi.org/10.1109/MS.2018.2801546] 
[38] Guo Y, Liang C. Blockchain application and outlook in the banking industry. Financ Innov 2016; 2(1) [https://doi.org/10.1186/s40854-016-0034-9]

[39] Zetzsche DA, Arner DW, Buckley RP. Decentralized Finance (DeFi). SSRN Journal 2020 [https://doi.org/10.2139/ssrn.3539194]

[40] Chen Y. Blockchain tokens and the potential democratization of entrepreneurship and innovation. Business Horizons 2018; 61(4): 567-75 [https://doi.org/10.1016/j.bushor.2018.03.006]

[41] Poblet M, Allen DWE, Konashevych O, Lane AM, Diaz Valdivia CA. From Athens to the Blockchain: Oracles for Digital Democracy. Front. Blockchain 2020; [https://doi.org/10.3389/fbloc.2020.575662]

[42] Reinsberg B. Blockchain technology and the governance of foreign aid. J. Institutional Econ. 2019; 15(3) [https://doi.org/10.1017/S1744137418000462]

[43] Treiblmaier H, Beck R, editors. Business Transformation through Blockchain. Springer; 2019.

[44] Brosens T. Why Bitcoin is destined to become a niche asset: ING; 2017. Digital Currencies.

[45] Demirgüç-Kunt A, Klapper L, Singer D, Ansar S, Hess J. The Global Findex Database 2017: World Bank Group; 2018.

[46] Gu W, Raghuvanshi A, Boneh D. Empirical Measurements on Pricing Oracles and Decentralized Governance for Stablecoins. SSRN Journal 2020 [https://doi.org/10.2139/ssrn.3611231]

[47] Lipton A, Sardon A, Schär F, Schüpbach C. From Tether to Libra: Stablecoins, Digital Currency and the Future of Money; 2020.

[48] El Faqir Y, Arroyo J, Hassan S. An overview of decentralized autonomous organizations on the blockchain. In: Robles G, Stol K-J, Wang X, editors. An overview of decentralized autonomous organizations on the blockchain; 2020. ACM; 1-8.

[49] Rozas D, Tenorio-Fornés A, Díaz-Molina S, Hassan S. When Ostrom Meets Blockchain. SAGE 2021; 11(1) [https://doi.org/10.1177/21582440211002526]

[50] Cuende L. Twitter Threat; 2020. Available from: URL: twitter.com/licuende/status/1263511552709267456.

[51] Buterin V. Ethereum Whitepaper: Ethereum; 2013.

[52] Jentzsch C. Decentralized Autonomous Organization to Automate Governance; 2017.

[53] Chohan UW. The DAO and Governance Issues. SSRN Journal [https://doi.org/10.2139/ssrn.3082055]

[54] Hassan S, Filippi P de. Decentralized Autonomous Organization. Internet Policy Review 2021; 10(2) [https://doi.org/10.14763/2021.2.1556]

[55] Davidson S, Filippi P de, Potts J. Economics of Blockchain. SSRN Journal 2016 [https://doi.org/10.2139/ssrn.2744751]

[56] Treiblmaier H. Toward More Rigorous Blockchain Research: Recommendations for Writing Blockchain Case Studies. Front. Blockchain 2019; 2 [https://doi.org/10.3389/fbloc.2019.00003]
[57] Taskinsoy J. Bitcoin: The Longest Running Mania Tulips of the 21st Century? SSRN Journal 2019 [https://doi.org/10.2139/ssrn.3505953]

[58] Miscione G, Ziolkowski R, Zavolokina L, Schwabe G. Tribal Governance. HICSS [https://doi.org/10.24251/HICSS.2018.566]

[59] Maker Foundation. The Maker Protocol: MakerDAO's Multi-Collateral Dai (MCD) System; 2020.

[60] Maker Foundation. MakerDAO Documentation; 2021 [cited 2021 January 25] Available from: URL: https://docs.makerdao.com/.

[61] Maker Foundation. Maker Protocol 101; 2020.

[62] Maker Foundation. Developer Guides and Tutorials; 2021 [cited 2021 January 25] Available from: URL: https://docs.makerdao.com/mcd-developerguides/developer-guides-and-tutorials.

[63] Maker Foundation. MakerDAO MCD FAQs; 2021. Available from: URL: https://communitydevelopment.makerdao.com/en/faqs.

[64] Maker Foundation. MakerDAO Forum; 2021 [cited 2021 January 25] Available from: URL: https://forum.makerdao.com/.

[65] The Authors. The De-Central Bank in Decentralized Finance: A Case Study of MakerDAO: Online Appendix [https://doi.org/10.5281/zenodo.5457246]

[66] Arnold L, Brennecke M, Camus P, et al. Blockchain and Initial Coin Offerings: Blockchain's Implications for Crowdfunding. In: Treiblmaier H, Beck R, editors. Business Transformation through Blockchain. Springer 2019; 233-72.

[67] Guggenberger T, Schlatt V, Schmid J, and Urbach N. A Structured Overview of Attacks on Blockchain Systems. PACIS 2021 Proceedings 2021.

[68] Maker Foundation. Auction Keepers; 2021 [cited 2021 February 2] Available from: URL: https://docs.makerdao.com/keepers/auction-keepers.

[69] Schellinger B. Optimization of special cryptocurrency portfolios. JRF 2020; 21(2): 127-57 [https://doi.org/10.1108/JRF-11-2019-0221]

[70] Maker Foundation. Governance Risk Framework; 2021 [cited 2021 February 2] Available from: URL: https://community-

development.makerdao.com/en/learn/governance/gov -risk-framework.

[71] Maker Foundation. MKR Token; 2021 [cited 2021 February 2] Available from: URL: https://communitydevelopment.makerdao.com/en/learn/governance/mkr -token.

[72] Maker Foundation. Introducing Governance; 2020. Available from: URL: https://communitydevelopment.makerdao.com/en/learn/governance/.

[73] Fridgen G, Radszuwill S, Schweizer A, Urbach N. Blockchain Won't Kill the Banks. Commun. AIS (forthcoming).

[74] Dyhrberg AH. Bitcoin, gold and the dollar - A GARCH volatility analysis. Finance Res. Lett. 2016; 16: [https://doi.org/10.1016/j.frl.2015.10.008]

[75] Jang S, Kim JY. A Study on The Asset Characterization of Bitcoin. J Soc e-Bus Stud. 